\title{
A Framework for Sensor Fusing Using Fuzzy Logic for Navigation Systems
}

\author{
Wong Wei Kitt \\ Ali Chekima \\ School Of Engineering and Information Technology \\ Universiti Malaysia Sabah \\ Locked Bag No. 2073 \\ 88999 Kota Kinabalu, \\ Sabah.
}

\begin{abstract}
Navigational sensors are evolving both on a commercial and research level. However, the limitation still lies in the accuracy of the respective sensors. For a navigation system to reach a certain accuracy, multi sensors or fusion sensors are used. In this paper, a framework of fuzzy sensor data fusing is proposed to obtain an optimised navigational system. Different types of sensors without a known state of inaccuracy can be fused using the same method proposed. This is demonstrated by fusing compass/accelerometer and GPS signal. GPS has evolved as a choice of navigation method for outdoor autonomous system. Despite it emerging trend and application, one problem remains is that it is still prone to inaccuracies due to environmental factors. These factors are available and evaluated in the GPS receiver architecture. These inaccuracies are available in the extracted NMEA(National Maritime Electronics Association) protocols as SNR (Signal to noise ratio) and HDOP (Horizontal Dillusion of Precision). Dead reckoning sensors on the other hand does not depend on external radio signal coverage and can be used in areas with low coverage. Unfortunately, the errors are unbounded and has an accumulative effect over time.
\end{abstract}

\section{Related works}

The emergence of maritime, aeronoutical technologies and autonomous system has led to a more demanding and accurate navigational system. Standalone navigational systems such as GPS (global positioning systems) and INS (Inertial measurement systems) has its respective limitations in application. Global positioning system works on constant updates from the GPS satelites. The faint

\author{
Jamal A. Dhargam \\ Farrah Wong \\ Tamer A.Tabet
}

School Of Engineering and Information Technology

Universiti Malaysia Sabah

Locked Bag No. 2073 ,

88999 Kota Kinabalu, Sabah

signals that are received by the receivers are often reflected by buildings causing a multi-path effect. Morever, in an urban environment, the LOS (Line of sight) of receivers and sattelites are often blocked by skyscappers and other concrete obstacles. This causes varying level of accuracies over time.

Inertial measurements units (IMU) are an alternative to the GPS system which works on acquiring the dynamics and acceleration of the platform to calculate the displacement of the platform. Unlike the GPS system, it doesn't receive any signals from external source and are prone to hardware errors that build up over a period of usage. Morever, the displacement calculated might not be as accurate in low velocities.

Due to the limitations of both sytems, many researchers were focused on fusing the several types of sensors. Many researcher are working on sensor fusion methods.

One classical approch is by using Kalman filter. One example is cited in [1] which uses the classical kalman filter for GPS/INS data fusing. In [2] and [3], several other data fusing methods were cited, among others using artificial intelligence algorithm such as fuzzy logic and neural network. On the application level, many UAV projects employs fusion tecniques for a more accurate UAV system and can be found both in [8] and [4].In these research works, the frame fixed coordinate is converted into earth fixed coordinate using DCM (Direction Cosine Matrix). For navigation that involves a wheeled platform, the odometry system were also included in the navigation 
system and fused together with the GPS/INS system. This method is applied in [9] using kalman filters. Several other navigation methods that involves image flow to estimate the velocity, and gyrocopic telemetry data are currently being developed for UAV and other autonomous systems.

\section{Quick view on GPS and INS system}

Navigation can be defined as the method of locating and tracking a certain coordinate. In order for a system to perform navigation, it has to be abled to accurately determine its own position. Currently, two systems were widely used for navigation. Both system have have their own limitations and advantages in terms of applications. GPS (Global Positioning System) receives constant radio signals from orbiting sattelites. From these signals, the GPS receiver figures out its position by comparing its distance from the sattellites. This provides constant updates from the GPS sattelites. However the GPS system has fluctuating quality of receiving data which is refered to as GPS 'outages.' The faint radio signals needs a line of sight from the sattelites for an acceptable signal. Similar to radio signals, the atmospheric condition can greatly impact the signal quality. This degradation of signal quality due to atmospheric conditions is known as the the Signal to noise ratio(SNR). Apart from the SNR, the quality of the the GPS data can also be effected by the geometry of the position of sattelites. The sattelites forms a tetrahedron shape that enables the receiver to have a three dimentional position tracking (latitude, longitude and attitude). Angle and geometry of this tethraheron also effects the accuracy. This element is known as Dilution of Precision (DOP). Lastly, the number of detectable Sattelites can also effect the GPS reading. The more detectable Satelites within line of sight range, the more accurate the GPS reading. GPS Coordinates given are Earth fix coordinate system which means that the coordinates given follow the axes that rotate with the earth rotation. Information on GPS data validity and the respective outages are explained in detailed in [6] and [7].INS navigation system (INS) are also known as dead reckoning navigations. This navigation system doesn't receive any updates from external sources. INS navigation system measures the acceleration of the frame fixed axis and calculates the displacement by intergating the acceleration values. Unlike the GPS navigation system, the INS navigation system have unbounded errors which means that the error in position is accumulated over time. Moreover the errors are also intergrated hence creating a amplified errors in navigation. The advantage of the INS navigation system is that it can be operated in covered area where there is no line of sight range to the satellites.

Hence,the fusion of these two navigation systems complement the respective weaknesses. In area where there is low GPS signal, the INS can serve as the prime navigation system whereas in areas with acceptable GPS signal, the GPS can serve as the primary navigation update and also recalibrate the accelerometer systems.

Fuzzy Logic was developed by Lofti A. Zadeh in [5]. The concept of fuzzy logic uses vague verbal expressions for processing. The crisp value are converted to a heuristic value . In contrast to the conventional model based systems, the heuristic values are processed using a set of If -Then Logics.

\section{Hardware development}

The application of fuzzy logic in fusing sensors is demonstrated by fusing the accelerometers and GPS. This is a classical aproach that has been in many comercial smartphones using kalman filter.The hardware for this hybrid navigation system consist of a GPS receiver ,three axis accelerometers and a digital compass. The. Figure 1 shows the block diagram of the hardware system. All the sensors are intergrated to a single microcontroller via ADC interface and transmitted to the processor via serial interface and evaluated using matlab fuzzy logic toolbox. Figure 1 shows the block diagram of the hardware setup and figure 2 shows the prototype of the hardware.

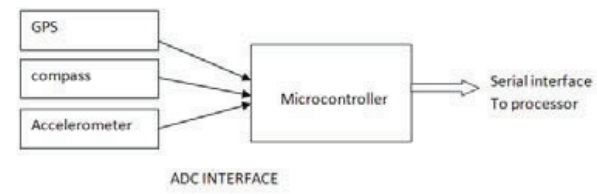

Figure 1: Hardware developement for the hybrid system 

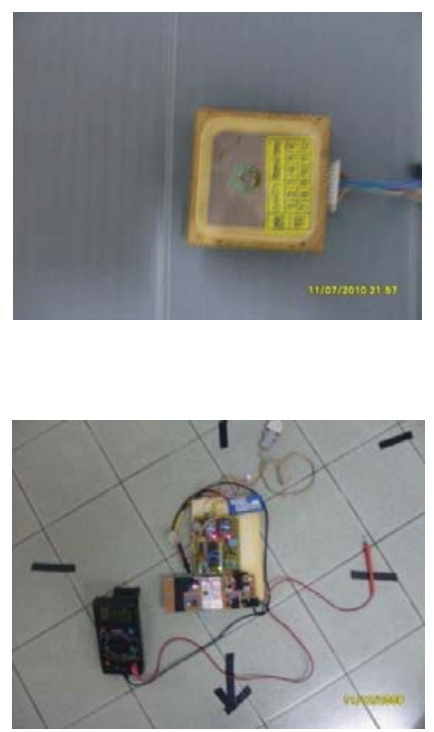

Figure 2 :GPS receiver intergrated to a microcontroller based circuit.

IV. Sensor fusing with GPS/Accelerometers The overall system of the hybrid GPS/INS can be seen from figure 2 . The section highlighted in the 'blue' boundary- GPS receivers provide the GPS latitude and longitude which are the earth axis fixed coordinate (not to be confused with Earth cartesian cordinate system). The Compass provide the angle of the velocity and the accelerometers provides the forward velocity $V f$ from equation (1)

$$
V f=\sqrt{v_{x}^{2}+v_{y}^{2}+v_{z}^{2}}
$$

where $v_{x}, v_{y}, v_{z}$ are the component velocity along the $\mathrm{x}, \mathrm{y}$ and $\mathrm{z}$ axis on the frame fixed axis.

The $V f$ is an aproximation of the actual forward velocity by neglecting the tilt angle.The accelerometer navigation block converts the accelerometer to travelled distances via a double intergration of the measured acceleration as shown in the equaltion in (2)

$$
D=\iint_{t=0}^{t=k} x d a d v
$$

where $\mathrm{D}$ is the distance traveled and $\mathrm{a}$ is acceleration values.

In many circumstances, the accelerometer requires recalibration when it is at static mode. Examples of these was mentioned in [7] and [8]. The speed of the accelerometers are constantly updated using the acceleration from the GPS while the bearing is constantly updated from the Compass. The distance travelled is given by the angle of heading multiplied by the distance travelled over the bounded period as shown in (3) and (4).

$$
\text { Latitunal distances }\left(\mathrm{D}_{\mathrm{lat}}\right)=\sin \emptyset \times \int_{t=k-1}^{t=k} V f+c d D
$$

Longitunal distances $\left(\mathrm{D}_{\text {lon }}\right)=\cos \emptyset \times \int_{t=k-1}^{t=k} V f+c d D$

where $\mathrm{D}$ is the distance traveled, $\varnothing$ is the angle bearing and $V f$ is the forward velocity, $\mathrm{c}$ is the previous calculated velocity.

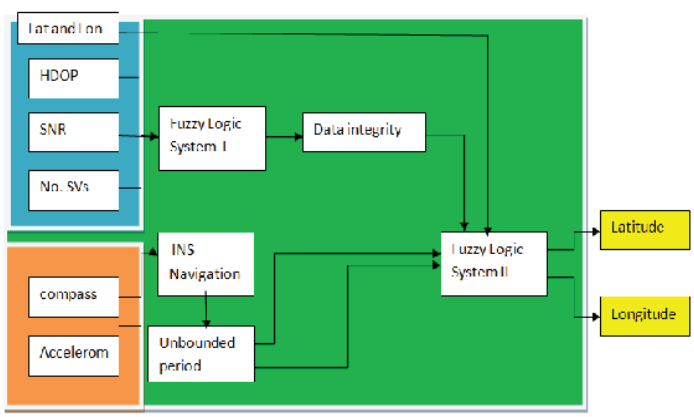

Figure 3: Block diagram of navigation architecture

The fuzzy logic system I (refer to Figure 1) to evaluate the GPS coordinates returns a value of data integrity for evaluation. As can be seen from figure 3 , The respective value that shows the outages of the GPS are the HDOP (Horizontal dilution of precision), SNR (Signal to noise ratio) and no. of SVs (no. Detectable sattelites in range). These values are 'fuzzified' into verbal values are shown in the membership function shown in figure 4,5, and 6.

Generally if the input function shows a high level of GPS outages at any of the input membership function, an overall 'low quality' will be output at the output membership function. If all the input membership displays an average level of GPS outages, the output membership function will consequently give an average quality of the GPS data.

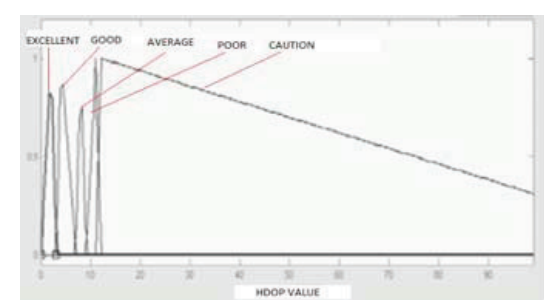

Figure 4 : Membership function for the HDOP Values 


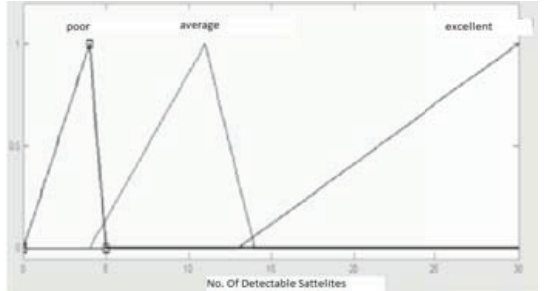

Figure 5: Membership function for the no. of SVs Values

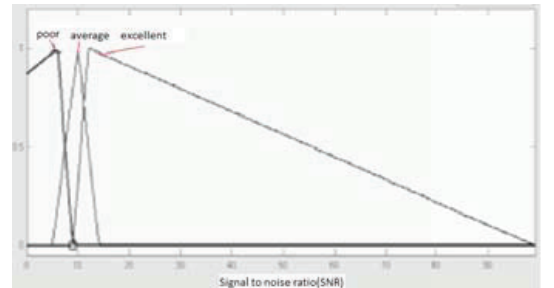

Figure 6 : Membership function for the SNR Values

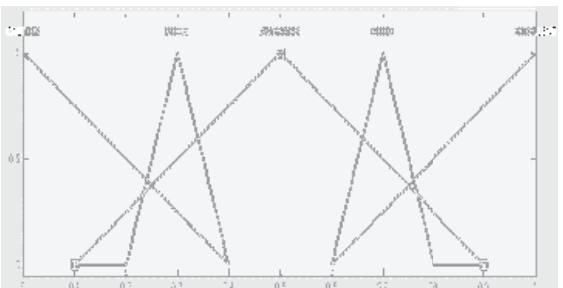

Figure 7 : Output Membership function for the SNR Values

The fuzzy logic II module (refer to figure 3) evaluates and decides the sensor that will give the input to predict the Position. The input membership functions are the Evaluated GPS data quality and period of dead reckoning without update from validated GPS data. The output membership function is the decision to use the data from GPS or accelerometer/compass. The block diagram for the fuzzy system is shown is figure 8 .

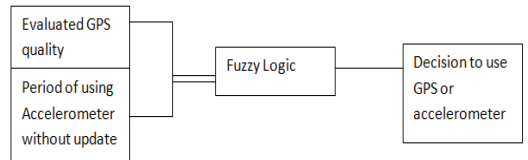

Figure 8: Block diagram of the fuzzy system

The rule base of the fuzzy system is as below: If GPS data is not valid, then Accelerometers data are used

If GPS data is valid, then GPS data are used

If period of using accelerormeter is long without update from GPS and GPS Data is unvalid, then accelerometer reading is used
As can be seen from the rule base, the accelerometer data is still prefable despite long period without update in the presence of low data validity of GPS data.The output membership is a sliding value from 0 to 1 . A value nearer to 0 meaning the accelerometer is more prefable and value nearer to 1 meaning the GPS is more prefable. A scenario of a certain navigation and localization process can be seen from figure 9 . showing the time frame of operation in seconds.

At $\mathrm{t}=0$, The GPS gives a valid data input. The first fuzzy logic would output a low GPS validity hence validating the information given. Based on this, the second fuzzy logic outputs a 1 -Meaning that the GPS data should be used. At $\mathrm{t}=1$ to $\mathrm{t}=5$, the platform passes through an area with densed concrete coverage hence causing the GPS receiver to have high level of GPS outages/low level of validity. From this, the second fuzzy logic will give an output of less than 0.5 meaning the accelerometers are the prefable navigation sensor over GPS receiver. The period of absense without valid GPS update is 5 seconds.

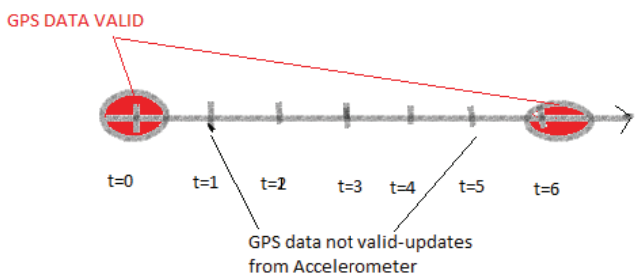

Figure 9:Time line of navigation

\section{Preliminary results}

The GPS/INS system is tested on a tunnel as shown in figure 10.The concrete covering blocks any LOS to the GPS sattelite causing absence of GPS signal in position 2 and position 3.(Refer to figure 11) Result show that the GPS/INS fussion system shows the updating of navigation coordinates using accelerometer despite the absense of GPS signal .The LLS (Lat Lon Altitude)coordinates are converted to earth measurements in order to synchronize both accelerometer and GPS data. The methods are explained in [11]. In future phases of the research, more readings will be carried in venues with varying degrees of GPS data validity. 


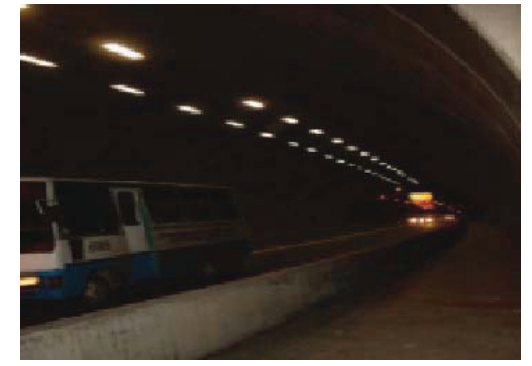

Figure 10: Tunnel used for preliminary testing

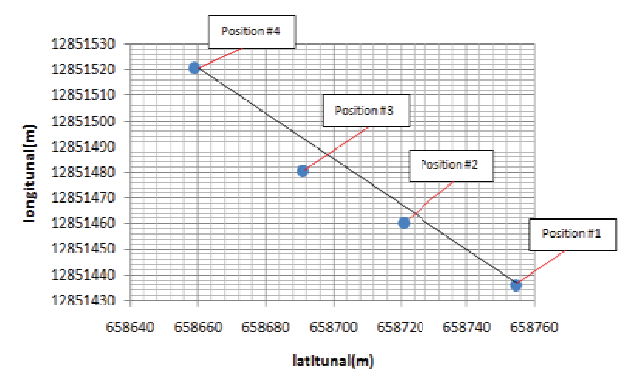

Figure 11 :Path of tracked points

The fuzzy logic Accelerometer/compass hybrid have demonstrated the the application of fusing sensors with known states of inaccuracy. These hybrids systems can be applied in autonomous systems that require navigations in places with low GPS sattelite coverage. Thus far, the hybrid system can only be used in areas with level plane. In the future, the system will be intergrated with a tiltmeter. Hence, the accelerometers can be compensated with the tilt angle.

$$
\text { Actual velocity }=V f \cos \emptyset
$$

where $\emptyset$ is the tilt angle and $V f$ is the forward velocity.

\section{Conclusion and future works.}

With the fuzzy logic system, countless navigational devices can be fuzed together for a more comprehensive navigation. For example vision flow system which has been developed in [10] way back in 1996 which enables the estimate of velocity in a certain platform. This can be fused together with the GPS/INS system for a more accurate navigation system.

In conclusion, we are constantly having new types of navigation sensors with known varying degree of accuracy. With the method proposed, these data can be fused for a more accurate navigation. The application of fuzzy logic controls presents an 'knowledge based alternative' to fuse these sensors with varying degree of inaccuaracies as shown in the block diagram in figure 12 .

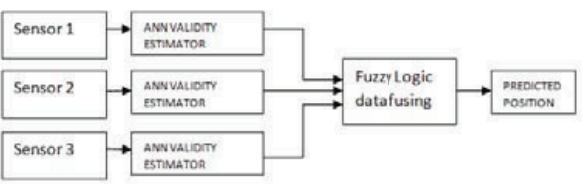

Figure 12: Block diagram of data fusing

For the GPS/INS system, the data is validated from the GPS data itself and the fuzzy logic. However, a neural network can be trained to predict the level of validity of respective sensors by considering the states that effect the accuracy of the sensors. The fuzzy logic system decides the sensor that has the highest level of validity for coordinate prediction. In the future phases of this research, sensors with high level of validity fluctuations can be intergrated together as long as the states that effect the inaccuracy of the sensors are known.

\section{References}

[1] David Mcneil Mayhew, May 1999, 'Multirate Sensor fusion for GPS Navigation using kalman Filtering', Masters' thesis, Virginia Polytecnic Institute and State University.

[2] Ahned M.Hassan, Khairulmizan Shamsuddin, Abdul Rahman Ramli, Raja Shamsul Azman, Salam A.Ismaeel, 2009, 'A review of navigation systems',Australian Journal of Basic and applied sciences.

[3] N.C Tsourveloudis, L.Doitsidis, K.P Valonis,'Autonomous Navigation of unmanned Vehicles:A fuzzy Logic perspective

[4] Chris Kreucher, Michael Beauvais , 'Using Fuzzy Logic for Autonomous navigation ", Department of Electrical and Computer Enginnering, University of Michigan.

[5] L.A.Zadeh,1965, 'Fuzzy Sets', Information and Control, 338-353(1965), Department Of Electrical and Electronics Research Laboratory, University Of California, Berkeley, Califonia

[6] Richard B. Langley, 'Dilution Of Precision', GPS World 1999, University of New Brunswick

[7] Laurie Tatleyand David Calcutt , 2003, 'Electronics Navigation Systems', ButterWorth Heinemann Publications, 
[8] Jong Hyuk Kim, Stuart Wishart and Sallah Sukannah, 2008, 'Real time navigation. Guidance and control of a UAV using Low cost sensors ', Australia Center for Field Robotics

[9] Cui Pingyuan, Xu Tianlai, 2007,' Data Fusion Algorithm for INS/GPS/Odometer Integrated Navigation', System Industrial Electronics and Applications, 2007.

[10] Amidi .O, 1996, 'An autonomous VisionGuided Helicopter' ,Ph.d Thesis, Electrical \& Computer Enginnering Dept. Carnegie Melon University

[11] http://www.csgnetwork.com/degreelenllav calc.html 